\title{
An unusual case of giant cell tumor of soft parts in an American Warmblood horse
}

\author{
Kurt Zimmerman ${ }^{1, *}$, Frederic Almy ${ }^{2}$, Geoff Saunders ${ }^{1}$, Mark Crisman ${ }^{3}$ and Leonardo Leonardi ${ }^{4}$ \\ ${ }^{1}$ Department of Biomedical Sciences \& Pathobiology, Virginia-Maryland College of Veterinary Medicine, Virginia \\ Tech University, Blacksburg, VA 24061, USA \\ ${ }^{2}$ Covance Laboratories Inc., Cincinnati, OH 45229, USA \\ ${ }^{3}$ Department of large Animal Clinical Sciences, Virginia-Maryland College of Veterinary Medicine, Virginia Tech \\ University, Blacksburg, VA 24061, USA \\ ${ }^{4}$ Department of Veterinary Medicine, University of Perugia, Perugia, Italy
}

\begin{abstract}
Fine needle aspiration and tissue biopsy samples were taken from a subcutaneous mass in the cranial stifle region of a 10-yr-old horse. Key cytologic features included numerous mildly to moderately pleomorphic histiocytic cells admixed with mononuclear inflammatory cells, occasional fibroblasts, multinucleated cells, and hemosiderin-laden macrophages. Based on these features, cytologic differentials included granulomatous inflammation with reactive fibroplasia, histiocytic sarcoma, giant cell tumor of soft parts (GCTSPs), and giant cell tumor of bone. The tissue biopsy supported a diagnosis of superficial GCTSPs. This neoplasm is of controversial origin but generally considered to be a soft tissue sarcoma and classified in the World Health Organization classification of tumors under fibrous histiocytic tumors as a giant cell tumor of soft tissue with low risk of malignancy. When found in the subcutis, this neoplasm rarely metastasizes in the horse and has a low rate of local reoccurrence. In this case, there was no local recurrence of the tumor after 6 mo, after which the horse was lost to follow-up.
\end{abstract}

Keywords: Cytology, Equine, Giant cell tumor of soft parts, Histopathology.

\section{Introduction}

The terminology, origin, and microscopic criteria related to giant cell tumors of animals have historically been confusing for both the veterinary clinician and pathologist. Part of this confusion is due to the fact that microscopic features of multinucleated macrophage-like giant cells can be seen in both nonneoplastic granulomatous inflammation and neoplasms of different cell lines (Pool and Thompson, 2002; Jo and Fletcher, 2014). Some examples of neoplasms that contain giant cells include giant cell tumor of bone (GCTb), histiocytic sarcoma, soft tissue sarcomas, osteoclastoma, and giant cell tumor of soft parts (GCTSPs) (May and Baker, 1985; Waters et al., 1994; Gonzalez-Campora et al., 1995; Perez-Martinez et al., 2000; Rodriguez-Peralto et al., 2001; Pool and Thompson, 2002). This confusion is worsened by the difference in biological behavior between these entities and even for the same neoplasm depending upon the species involved and site (Bush and Powers, 2008; Jo and Fletcher, 2014). In this paper, we report the occurrence of a GCTSP in a horse. We discuss some of the key clinical and microscopic features seen with this unique entity and provide a brief discussion of its origin and expected biological behavior. We hope that adding this case report to the body of literature will help decrease some of the confusion associated with GCTSP in horses.

\section{Case Details}

A 10-yr-old American Warmblood gelding used for event riding was examined by the referring veterinarian for a mild lameness of a few weeks duration. The clinician discovered a grade 1 lameness (American Association of Equine Practitioners guidelines) involving the left rear limb and a visible, firm, subcutaneous, non-painful swelling, $1 \mathrm{~cm} \times 8 \mathrm{~cm} \times 12$ $\mathrm{cm}$ in size involving the cranial stifle region of the same limb (Fig. 1). Radiographic examination did not show any bone changes in the region of the mass.

Fine needle aspirates were taken from the region and prepared as air-dried slides. The slides were submitted for cytologic evaluation to the Virginia-Maryland College of Veterinary Medicine (VMCVM) clinical pathology diagnostic service. The slides were Wright's stained and examined by a Diplomate of the American College of Veterinary Pathologists, Clinical Pathology (KZ, FA). Cytologic examination revealed a highly cellular sample with good cellular preservation. A pleomorphic population of individualized histiocytic cells predominated (Figs. 2-4). Mitotic figures were not seen. The histiocytic cells had indistinct cell borders and often formed multilayered clumps. 


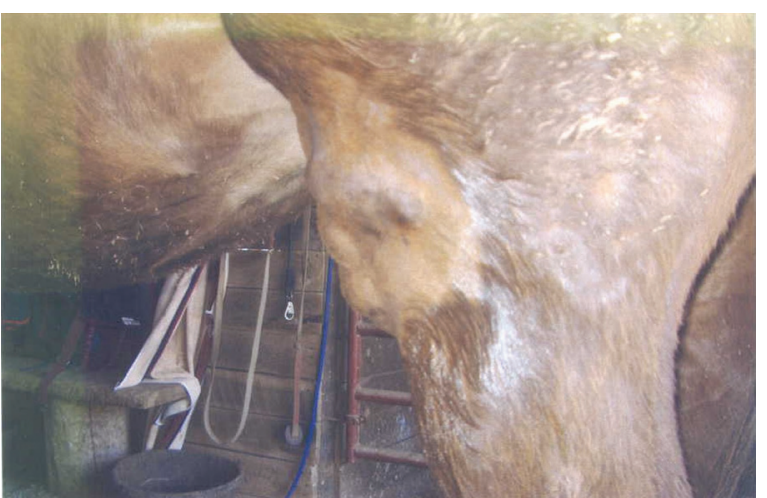

Fig. 1. Clinical appearance of a subcutaneous mass over the stifle region of a middle-aged American Warmblood gelding. Hair has been removed for visualization of the mass.

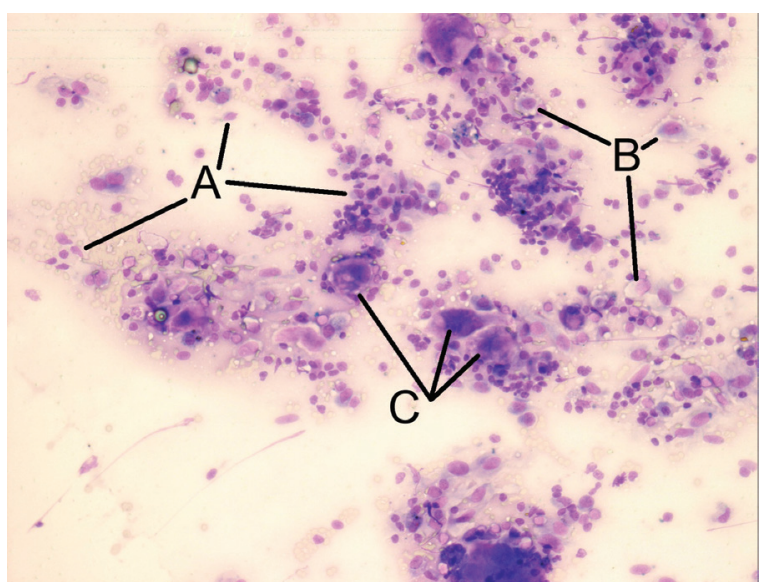

Fig. 2. Cytology sample from a subcutaneous mass on the rear limb of the horse showing loosely associated spindle (A) and histiocytic (B) cells mixed with multinucleated giant cells (C); Wright stain, 200×.

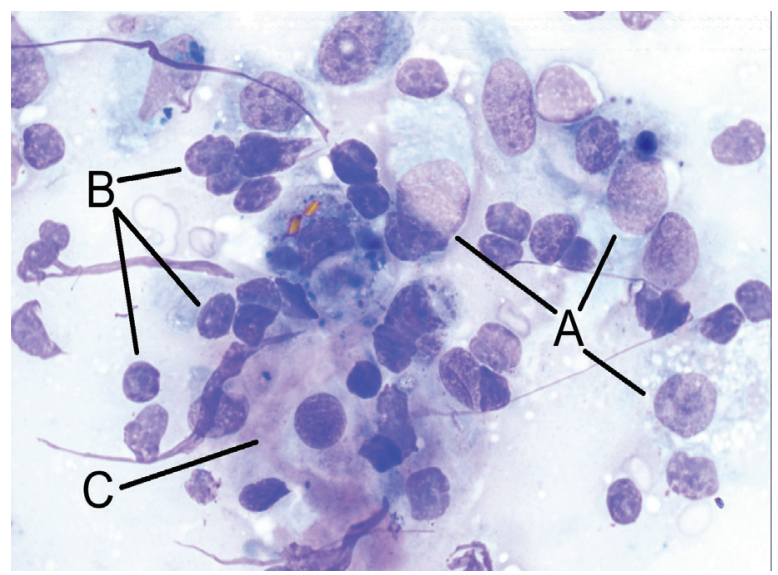

Fig. 3. Cytology sample from a subcutaneous mass on the rear limb of the horse showing pleomorphic histiocytic (A) cells with occasional dark pigment mixed with a few lymphocytes (B) and some amorphous extracellular matrix (C); Wright stain, 1,000×.

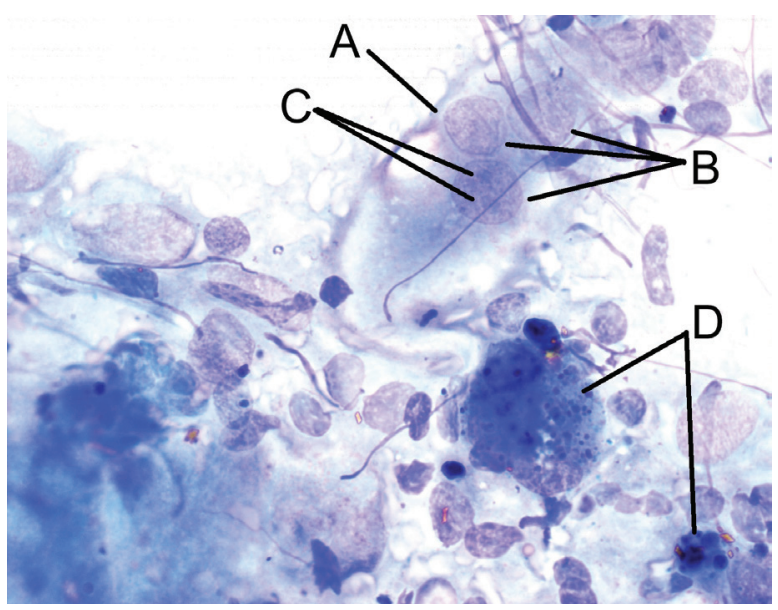

Fig. 4. Cytology sample from a subcutaneous mass on the rear limb of the horse showing the pleomorphic nature of the histiocytic cells (A), multinucleation (B), nuclear features of malignancy $(\mathrm{C})$ (multiple nucleoli of variable size) along with some dark cytoplasmic pigment (D); Wright stain, 1,000×.

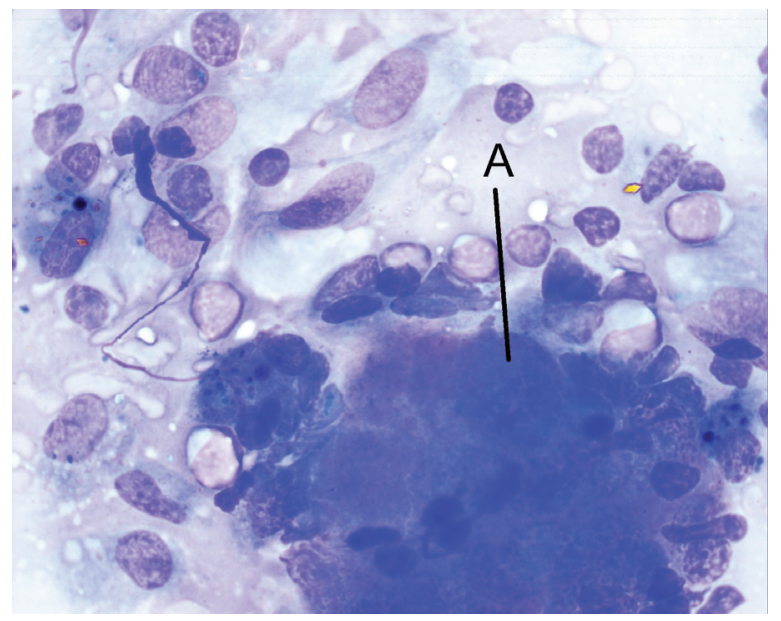

Fig. 5. Cytology sample from a subcutaneous mass on the rear limb of the horse showing atypia of multinucleated cells (A); Wright stain, $1,000 \times$.

Nuclei were oval and centrally located. Chromatin was coarsely stippled and contained multiple varioussized nucleoli. Anisokaryosis was marked in these cells and there was a small amount of extracellular matrix associated with some of the cells. Multinucleate cells containing up to 25-30 nuclei were also present (Fig. 5). Anisocytosis was moderate and the cytoplasm was homogenous and lightly basophilic. Admixed with the histiocytic cells were moderate numbers of small lymphocytes, occasional plasma cells, and rare nondegenerate neutrophils. Low numbers of larger bland spindle-shaped cells were also noted as were occasional erythrocytes and some lysed cells. Cytologic opinion was granulomatous inflammation with chronic hemorrhage and fibroplasia along with consideration 


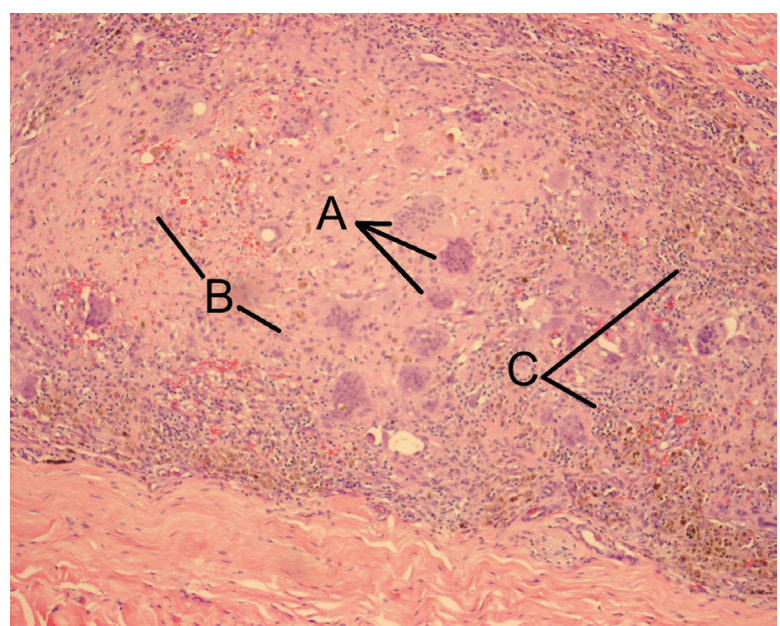

Fig. 6. Histologic tissue section from a subcutaneous mass on the rear limb of the horse showing an unencapsulated multinodular mass composed of giant cells (A) and histiocytic cells (B) with minimal stroma and some lymphoid aggregates (C); Hematoxylin and Eosin, 100×.

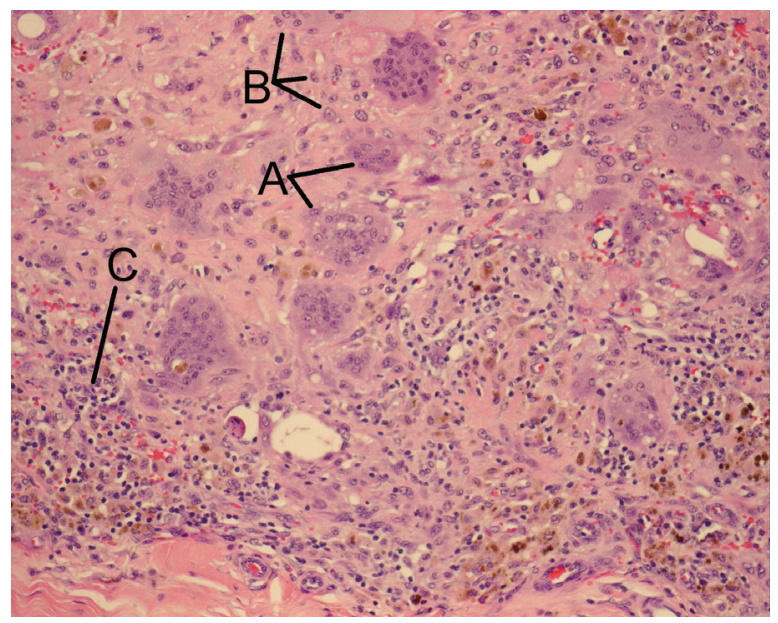

Fig. 7. Histologic tissue section from a subcutaneous mass on the rear limb of the horse, similar to Figure 6 but the higher magnification of multinodular mass composed of giant cells (A) and histiocytic cells (B) with minimal stroma and some lymphoid aggregates (C); Hematoxylin and Eosin, 200×.

for some form of soft tissue sarcomas such as histiocytic sarcoma, GCTSP, and GCTb.

Following cytology, the mass was taken from the horse while under general anesthesia by a total excisional surgical biopsy. The tissue was immediately fixed in neutral buffered $10 \%$ formalin and submitted for histopathologic processing at the VMCVM histopathology service. Slides were stained with Hematoxylin-Eosin and examined by a Diplomate of the American College of Veterinary Pathologists, Anatomic Pathology (GS) and by a veterinary pathologist (LL) specializing in bone tumors at the University of Perugia, Italy. Histopathologic

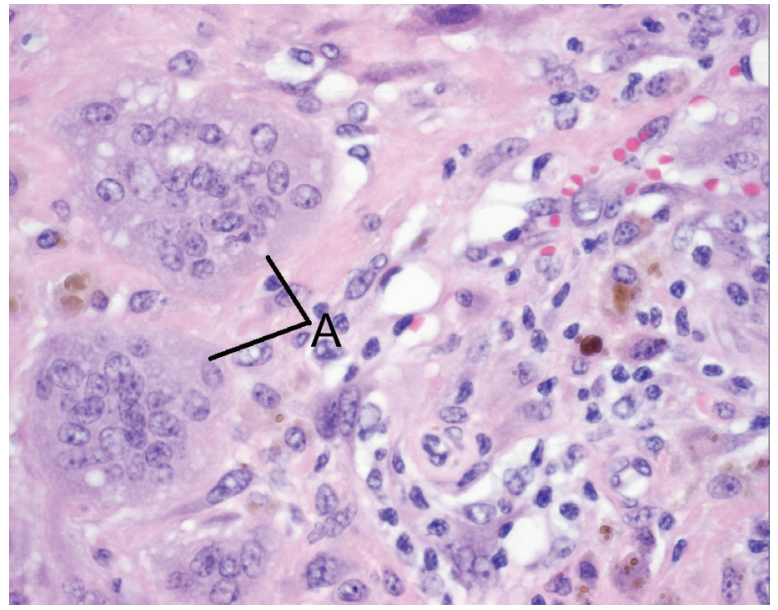

Fig. 8. Histologic tissue section from a subcutaneous mass on the rear limb of the horse showing features of multinucleated cells (A) within the mass; Hematoxylin and Eosin, 400×.

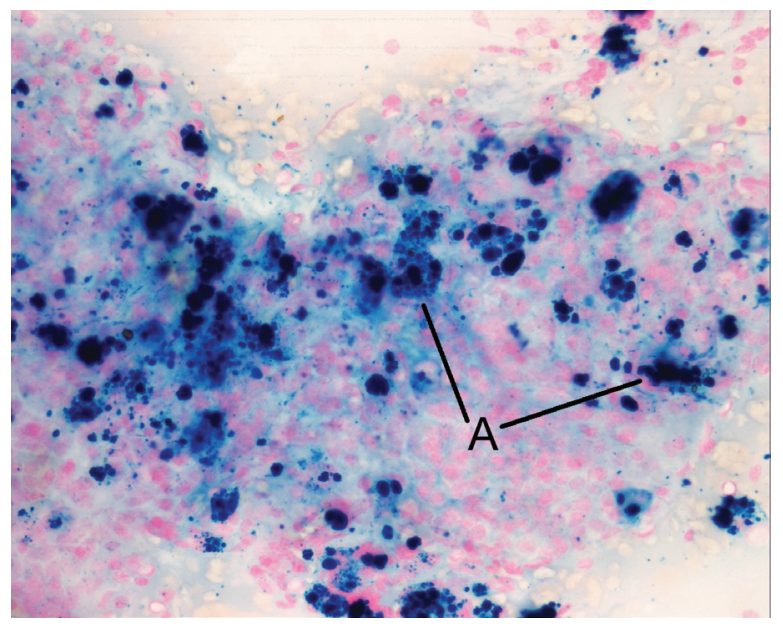

Fig. 9. Histologic section from a subcutaneous mass on the rear limb of the horse showing abundant iron pigment staining (A) with the parenchyma of the mass; Prussian Blue reaction, $400 \times$.

examination revealed an infiltrative but well delineated, encapsulated multinodular highly cellular mass composed of pleomorphic polygonal histiocytic cells supported by an abundant amount of fibrovascular stroma mixed with moderate numbers of multinucleate cells (Figs. 6-8). Mitoses were rare within the mass. Mantles of lymphocytes surrounded many of nodules. A moderate amount of hemosiderin and hematoidin pigment was detected with Prussia Blue reaction and seen scattered throughout the parenchyma of the mass (Fig. 9). The tentative histological diagnosis was GCTSP pending special stains to rule out infectious agents and supportive immunohistochemical results.

Histocytochemistry with Ziehl-Neelsen acid-fast, periodic acid-Schiff, and Gram staining were done using standard methods and no infectious agents were 
seen. Immunohistochemistry staining of paraffinembedded tissue sections of the mass along with positive and negative control tissues was done using a Ventana Autostainer (Ventana Medical Systems Inc., Tucson, AZ). The following immunostains were applied in conjunction with a red horseradish peroxidase marker (universal alkaline phosphatase red detection kit; Roche Inc., Basel, Switzerland): pan-keratin (epithelial; Roche Inc.), vimentin (mesenchymal; Roch Inc.), CD11c (leukocyte histiocytic; Leukocyte Biology Laboratory, University of California, Davis, CA), CD79a (lymphoid B-cell; Roche Inc.), CD3 (lymphoid T-cell; Roche Inc.), S100 (neuronal; Roche Inc.), smooth muscle actin (smooth muscle; Roche Inc.), and desmin (skeletal muscle; Roche Inc.). Most of the histiocytic and giant cells of the mass had strong cytoplasmic staining with vimentin and CD11c and occasional weak actin staining. These same cells did not stain with CD3, CD79a, S100, cytokeratin, or desmin. Lymphocytes within the mass were mostly CD3 positive. The immunohistochemical findings supported the histological diagnosis of GCTSP. At the 6-mo clinical recheck, there was no sign of local reoccurrence, after which the horse was lost for follow-up.

\section{Discussion}

Of the domestic species, cats are the most commonly affected with malignant GCTSP, but cases have also been reported in humans, dogs, horses, mules, pigs, cows, hamsters, and nonhuman primates (Ford et al., 1975; Hamir, 1989; Perez-Martinez et al., 2000; Pool and Thompson, 2002). In domestic animals, most cases occur in middle-age (mean 6.8, 6.5, and $8.4 \mathrm{yr}$ for horses, cats, and dogs, respectively) (Pool and Thompson, 2002). Females are slightly more at risk than males in dogs and humans (Pool and Thompson, 2002; Kim and Han, 2003). There appears to be a breed predilection for shorthaired cats. However, breed patterns are less clear for dogs and horses because of fewer reported cases (Ford et al., 1975; Render et al., 1983; Pool and Thompson, 2002). The extremities are the most likely site of occurrence and the hind limb is more commonly involved in the horse, and males may be at higher risk (Ford et al., 1975; Render et al., 1983; Hamir, 1989; Pool and Thompson, 2002; Marryatt et al., 2003; Bush and Powers, 2008; Cian et al., 2016). In a 2008 review of 21 GCTSP in horses, in spite of having a malignant histologic appearance, local reoccurrence following surgical removal and metastatic disease was rare (Bush and Powers, 2008).

While the origin of this neoplasm remains uncertain, in past veterinary literature, many considered it to be a subtype of malignant fibrous histiocytoma, which in itself a controversial diagnostic classification (Rodriguez-Peralto et al., 2001; Pool and Thompson, 2002; Marryatt et al., 2003; Cian et al., 2016). In the
World Health Organization classification of tumors for human's, superficial forms of this neoplasm are named giant cell tumor of soft tissue and categorized under fibrohistiocytic tumors (Jo and Fletcher, 2014). They are considered to have a low potential for malignancy, while malignant variants are classified as undifferentiated/pleomorphic sarcoma with giant cells under undifferentiated/unclassified sarcoma (Jo and Fletcher, 2014; Cian et al., 2016). Regardless of the nomenclature used to identify this neoplasm, given the common positive immunohistochemical staining pattern of vimentin and CD11c, GCTSPs in horses are likely to be giant cell-rich type sarcoma of mesenchymal origin (Bush and Powers, 2008; Jo and Fletcher, 2014; Cian et al., 2016).

In horses, the neoplasm occurs most commonly as a multinodular, subcutaneous mass in proximity to one of the larger joints of the rear limb, but it has also been reported in other sites (neck and shoulder) (Render et al., 1983; Bush and Powers, 2008). Microscopically, the neoplasm is composed of large multinucleate giant cells (2-150 nuclei), pleomorphic histiocytic cells, fibroblasts, and spindle cells with mild anaplastic features. The mitotic rate is low (Ford et al., 1975; Render et al., 1983; Hamir, 1989; Pool and Thompson, 2002; Bush and Powers, 2008; Cian et al., 2016).

Benign giant cell tumor of tendon sheath (BGCTOTS) is another well-recognized entity in veterinary medicine and likely represents a variant of GCTSP (Ford et al., 1975; Gonzalez-Campora et al., 1995; Pool and Thompson, 2002). BGCTOTS have been reported in cats, dogs, and rarely in horses with some overlap in occurrence sites for GCTSP (Pool and Thompson, 2002). However, others are of the opinion that BGCTOTS represents a manifestation of a localized nodular or villonodular synovitis, a condition resulting from hypertrophy of the joint capsule in response to chronic inflammation or a low-grade neoplasm (Gonzalez-Campora et al., 1995; Pool and Thompson, 2002). Interestingly, a common feature of BGCTOTS in both horses and humans is the presence of hemosiderin-laden macrophages, a feature which was very prominent in the cytology and histopathology specimens of this case (Fig. 6) (Pool and Thompson, 2002; Kim and Han, 2003; Bush and Powers, 2008; Cian et al., 2016).

From a cytopathologist's perspective, this case demonstrates the challenge of differentiating granulomatous inflammation from GSTSP in the horse. Two clinical pathologists independently evaluated samples from this case and arrived at a similar interpretation of granulomatous inflammation with suspicion for histiocytic sarcoma. The lack of an etiologic agent, mildly to moderately pleomorphic histiocytic cells admixed with mononuclear inflammatory cells, occasional fibroblasts, and multinucleate cells, and numerous hemosiderin-laden 
macrophages were the key cytologic features noted in this case. Given the microscopic feature overlap between these two entities, caution is advised in arriving at diagnosis solely based on cytology results alone. In addition, other differentials to be considered in horse with similar cytologic and clinical findings should include sarcoid, scar tissue, foreign body reaction, fibroma or fibrosarcoma, lipoma or liposarcoma, leiomyoma or leiomyosarcoma, synovial cell tumor, and osteoclastoma (GCTb) (Leonardi et al., 2013; Marryatt et al., 2003). In this case, the clinical location of the mass along with the histopathology features and positive immunohistochemical staining with vimentin and CD11c supported the diagnosis of GSTSP. This report adds to the growing veterinary literature on GCTSP and helps remind attending clinicians and pathologists of the possibility of this unusual soft tissue neoplasm with a predilection for occurrence in the rear limb of horses .

\section{Conflict of interest}

The authors declare that there is no conflict of interest.

\section{References}

Bush, J.M. and Powers, B.E. 2008. Equine giant cell tumor of soft parts: a series of 21 cases (20002007). J. Vet. Diagn. Invest. 20, 513-516.

Cian, F., Whiteoak, S. and Stewart, J. 2016. A case of giant cell tumor of soft parts in a horse. Vet. Clin. Pathol. 45, 501-504.

Ford, G.H., Empson, R.N., Jr., Plopper, C.G. and Brown, P.H. 1975. Giant cell tumor of soft parts. A report of an equine and a feline case. Vet. Pathol. $12,428-433$.

Gonzalez-Campora, R., Salas Herrero, E., OtalSalaverri, C.V.-R.J.L., San Martin Diez, V., Hevia Vazquez, A. and Galera Davidson, H. 1995. Diffuse tenosynovial giant cell tumor of soft tissues. Report of a case with cytologic and cytogenetic findings. Acta Cytol. 39, 770-776.

Hamir, A.N. 1989. Equine giant cell tumor of soft tissues. Cornell Vet. 79, 173-177.

Jo, V.Y. and Fletcher, C.D. 2014. WHO classification of soft tissue tumours: an update based on the 2013 (4th edition). Pathology 46, 95-104.
Kim, N.R. and Han, J. 2003. Primary giant cell tumor of soft tissue. Report of a case with fine needle aspiration cytologic and histologic findings. Acta Cytol. 47, 1103-1106.

Leonardi, L., Quattrini, I., Roperto, F. and Benassi, M.S. 2013. Protease expression in giant cell tumour of bone: a comparative study on feline and human samples. Res. Vet. Sci. 95, 310-315.

Marryatt, P.A., Folpe, A.L., Morris, R.J., Weiss, S.W., Render, J.A., Harrington, D.D., Wells, R.E., Dunstan, R.W., Turek, J.J., Boosinger, T.R., Ford, G.H., Empson, R.N., Jr., Plopper, C.G. and Brown, P.H. 2003. Malignant giant cell tumor of soft parts in a mare. Can. Vet. J. 44, 743-748.

May, S.A. and Baker, J.R. 1985. A giant cell tumour (osteoclastoma) of the tibia in a horse. Equine Vet. J. 17, 477-479.

Perez-Martinez, C., Garcia Fernandez, R.A., Reyes Avila, L.E., Perez-Perez, V., Gonzalez, N. and Garcia-Iglesias, M.J. 2000. Malignant fibrous histiocytoma (giant cell type) associated with a malignant mixed tumor in the salivary gland of a dog. Vet. Pathol. 37, 350-353.

Pool, R.R. and Thompson, K.G. 2002. Tumors of joints. In Tumors in domestic animals. Ed., Meuten, D.J. Ames, IA: Iowa State University Press, pp: 199317.

Render, J.A., Harrington, D.D., Wells, R.E., Dunstan, R.W., Turek, J.J. and Boosinger, T.R. 1983. Giant cell tumor of soft parts in six horses. J. Am. Vet. Med. Assoc. 183, 790-793.

Rodriguez-Peralto, J.L., Lopez-Barea, F. and Fernandez-Delgado, J. 2001. Primary giant cell tumor of soft tissues similar to bone giant cell tumor: a case report and literature review. Pathol. Int. 51, 60-63.

Waters, C.B., Morrison, W.B., DeNicola, D.B., Widmer, W.R. and White, M.R. 1994. Giant cell variant of malignant fibrous histiocytoma in dogs: 10 cases (1986-1993). J. Am. Vet. Med. Assoc. 205, 1420-1424. 